\title{
Diffusely scattered and transmitted elastic waves by random rough solid-solid interfaces using an elastodynamic Kirchhoff approximation
}

\author{
F. Shi ${ }^{\mathrm{a}, *}$, M. J. S. Lowe ${ }^{\mathrm{a}}$, R. V. Craster ${ }^{\mathrm{b}}$ \\ ${ }^{a}$ Department of Mechanical Engineering, Imperial College London \\ ${ }^{b}$ Department of Mathematics, Imperial College London
}

\begin{abstract}
Elastic waves scattered by random rough interfaces separating two distinct media play an important role in modelling phonon scattering and impact upon thermal transport models, and are also integral to ultrasonic inspection. We introduce theoretical formulae for the diffuse field of elastic waves scattered by, and transmitted across, random rough solid-solid interfaces using the elastodynamic Kirchhoff Approximation (KA). The new formulae are validated by comparison with numerical Monte Carlo simulations, for a wide range of roughness (rms $\sigma \leq \lambda / 3$, correlation length $\lambda_{0} \geq$ wavelength $\lambda$ ), demonstrating a significant improvement over the widely used small-perturbation approach which is valid only for surfaces with small rms values. Physical analysis using the theoretical formulae derived here demonstrates that increasing the rms value leads to a considerable change of the scattering patterns for each mode. The roughness has different effects on the reflection and the transmission, with a strong dependence on the material properties. In the special case of a perfect match of the wave speed of the two solid media, the transmission is the same as the case for a flat interface. We pay particular attention to scattering in the specular direction, often used as an observable quantity, in terms of the roughness parameters, showing a peak at an intermediate value of rms; this rms value coincides with that predicted by the Rayleigh parameter.
\end{abstract}

\section{Introduction}

Interface and surface roughness is well-known to significantly alter the elastic wave scattering field in solids Ogilvy (1991), and hence has considerable effects on ultrasonic applications Ogilvy and Foster (1989); Ogilvy and Culver5 well (1991); Pettit et al. (2015); Shi et al. (2016), and on boundary phonon scattering to model thermal transport in nanostructures Sadhu and Sinha (2011);

\footnotetext{
${ }^{*}$ Corresponding author.

E-mail address: f.shi12@imperial.ac.uk
} 
Carrete et al. (2011); Sun and Pipe (2012); Hertzberg et al. (2014); Maznev (2015); Maznev et al. (2015); on the atomic scale, the phonon-interface interaction can still be modelled by the macroscopic theory on elastic wave scattering in the long-wavelength limit Wen et al. (2009). These important applications have therefore motivated considerable work on rough surface scattering, however many studies are simply for acoustics, Sadhu and Sinha (2011); Hertzberg et al. (2014); Wen et al. (2009); Cuffe et al. (2013) that is, for solids omitting shear, and this is a substantial simplification as elastic solids support both

15 bulk shear and compressional waves. Moreover, these bulk waves have different wavespeeds and undergo mode conversion upon reflection/ transmission at an interface. An elastic stress-free surface also supports Rayleigh surface waves, and an interface supports Stoneley interfacial waves, and acoustic models fail to capture these: here we make no such simplification. There are other natural limits based upon the ratio of the dominant lengthscales in the problem, here the correlation length, the wavelength, and the root mean-squared (rms) height, and we shall concentrate here upon the situation where the correlation length is larger than the wavelength and then physically we can use the Kirchhoff approximation, but we shall not invoke any assumption of small rms values.

25 As MaznevMaznev (2015) notes, there is a heavy reliance upon numerical simulation, a lack of analytical results to draw upon, and open problems remain regarding scattering as a function of correlation length and/or roughness height.

Typical previous approaches study the scattering from a stress-free rough surface,rather than an interface separating two media, in which case the expected scattering is modelled from the knowledge of the statistics of the surface profile. The expected scattering in the specular direction, defined as the "specularity', is a widely used parameter to model the phonon transport through a rough interface, and this specularity significantly affects the efficiency of terahertz phonon devices Wen et al. (2009); Maznev (2015); Sun and Pipe (2012); Maznev et al. (2015). Many of the previous studies rely on using the coherent scattering intensity $I^{c}$ at the specular direction Ogilvy $(1986)$; Chen et al. (2008); Heron et al. (2009); Aksamija and Knezevic (2010); Cuffe et al. (2013); Hertzberg et al. (2014) as proposed, for instance, by Ogilvy Ogilvy (1991):

$$
\begin{aligned}
I^{c} & =I^{\text {flat }} \exp \left(-g_{\alpha \beta}\right), \quad \alpha, \beta=p, s \\
g_{\alpha \beta} & =\left(k_{\alpha} \cos \theta_{i}+k_{\beta} \cos \theta_{s}\right)^{2} \sigma^{2}
\end{aligned}
$$

where $I^{\text {flat }}$ is the scattering intensity from a flat surface, and ignore the dif30 fuse scattered field. In Eq. (1), $k_{\alpha / \beta}$ represents the wavenumber for incident/scattered waves, which can be either a compressional $(\mathrm{P})$ or shear $(\mathrm{S})$ mode. The wavenumber $k_{\alpha / \beta}$ equals to $\frac{\omega}{c_{\alpha / \beta}}$, where $c_{\alpha / \beta}$ refers to the wavespeed and $\omega$ is the angular frequency. The incidence and scattering angles are denoted as $\theta_{i}$ and $\theta_{s}$, and $\sigma$ is the rms value of the surface height. Many studies regarding 35 phonon boundary scattering have applied a simpler similar equation ascribed to Ziman Ziman (1960), although it does not include the elastic mode conversion and oblique incidence/scattering angles as Eq. (1) does. Eq. (1) considers only 


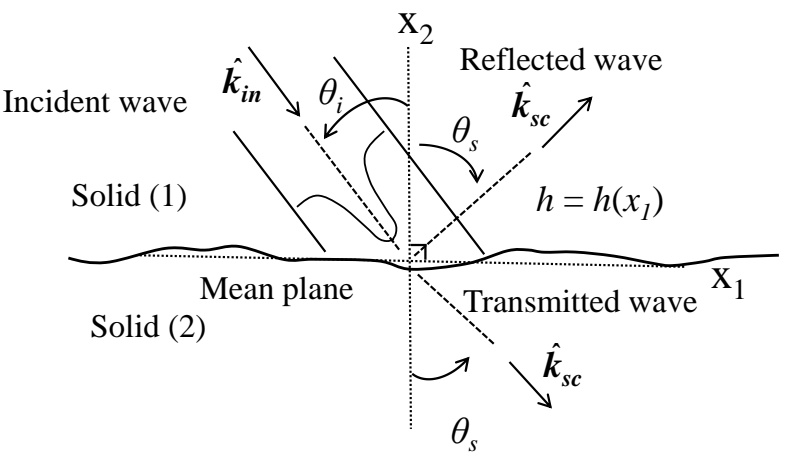

Figure 1: Sketch for an incident Gaussian beam ( $\mathrm{P}$ wave) scattered from a 1D rough interface separating two elastic solids.

the reflection case for a stress-free boundary condition (i.e. solid-air interface); the transmission case needs a different formula, which we derive in this article.

It is well established that Eq. (1) is only valid in the specular direction for surfaces with a very small roughness (e. g. $k \sigma \ll 1$ and $k \lambda_{0} \gg 1$ ). Additional contributions to the total scattering field from the diffuse waves, which sometimes are comparable with, or even dominant over, the coherent field, are not 45 included in the formula. The importance of the diffuse field has been highlighted by a number of reports Weber et al. (1978); Wen et al. (2009); Maznev (2015). Experiments Wang et al. (2011); Hertzberg et al. (2014) on the thermal transport in nanostructures have indicated that the use of Eq. (1) is often inconclusive and does not explain the experimental findings, requiring alternative

${ }_{50}$ theories to account for the diffuse field. In addition Maznev Maznev (2015) has shown that using Eq. (1) to calculate the specularity tends to overestimate the heat flux dissipation due to loss of the diffuse field.

To model the diffuse scattering from rough boundaries, it has been necessary to utilize sophisticated numerical techniques that have been developed recently, based around the finite-element (FE) method Pettit et al. (2015); Shi et al. (2014), the Green's function method Zhao and Freund (2009), and molecular dynamic model Liang et al. (2014). These numerical methods require considerable computation resources and it is not easy to generalize conclusions of how

60 the surface statistics affect the scattering beyond the specific cases simulated unless the statistics are gathered from many multiples of such simulations. Hence there is substantial motivation to develop analytical tools to tackle these problems.

Analytical methods have the advantage that they offer physical insight into the scattering behavior, provided the method is within their region of validity. 
A perturbation approach, assuming the roughness amplitude is small relative to wavelength, and surface slopes are also small, has been applied recently Sun and Pipe (2012); Maznev (2015), to study the effect of roughness and material

70 properties on the scattering of the diffuse field. However, such a perturbation theory is only valid for modelling scattering from weakly rough surfaces $(\sigma \leq \lambda / 10$ for the first-order approximation Thorsos and Jackson (1989)). One important question posed by Maznev Maznev (2015) concerns the possibility of finding a complementary theoretical method to model the scattering beyond

75 the validity of the perturbation approximation, for surfaces with median and high roughnesses: at high roughness (large rms value) the diffuse field dominates and the scattering is completely different from that with a weakly rough surface.

In this article we develop a theoretical method that is valid for surfaces with a wide range of roughness by using the elastodynamic Kirchhoff approximation (KA). The KA has been found to be accurate in modelling the scattering from surfaces with roughness up to $\sigma=\lambda / 3$, when the correlation length $\lambda_{0}$ is comparable with or larger than one wavelength Thorsos (1988); Shi et al. (2015). Despite the early application of KA in acoustics Thorsos (1988), it is only re85 cently that the elastodynamic KA has been developed to model theoretically the diffuse field from an elastic rough surface including mode conversions Shi et al. (2016); there are details in elasticity, the mode conversion at surfaces, two types of bulk waves, that prevent a direct generalization. In Shi et al. (2016) only a single medium is considered and the rough surface is assumed to be stress90 free, hence only allowing for reflection and preventing application of the theory to cases where transmission is important. In many situations, it is critical to consider two media and include the rough solid-solid interface between them, for instance in the study of the boundary phonon scattering/transport at the $\mathrm{Si} / \mathrm{Ge}$ interface commonly seen for a phonon device Sun and Pipe (2012). The 95 physics of solid-solid rough interface scattering can be very different from that of a single medium with a stress-free boundary condition. For instance, a considerable portion of incident energy can be transmitted through the interface, and the roughness could be expected to have different effects on the reflection and transmission depending on the materials; even the reflection itself would be different from that with a stress-free rough surface.

This article is organized as follows: Section 2 describes the derivation of the theoretical formulae representing the diffuse scattering field using the KA. Numerical simulations are performed in Section 3 to explore the accuracy of the derived expressions. Section 4 illustrates effects of roughness and material properties on the diffuse field, for different modes. Finally, we draw together concluding remarks in Section 5. 


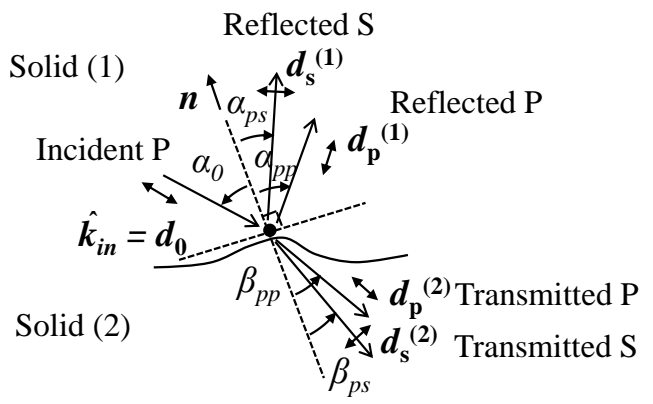

Figure 2: Illustration of the elastodynamic Kirchhoff approximation

\section{Theoretical formulae with Kirchoff approximation}

The scattering system in 2D is shown in Fig. 1, with a rough interface, separating two elastic solids, insonified by an incident $\mathrm{P}$ wave from Solid 1. A fraction of the elastic waves transmit through the interface to Solid 2, while the rest are reflected back into Solid 1. The unit incident and scattering vectors for $\mathrm{P}$ or mode-converted $\mathrm{S}$ waves are denoted as:

$$
\begin{aligned}
& \hat{\boldsymbol{k}}_{\boldsymbol{i n}}=\left(\sin \theta_{i},-\cos \theta_{i}\right) \\
& \hat{\boldsymbol{k}}_{\boldsymbol{s c}}=\left\{\begin{aligned}
\left(\sin \theta_{s}, \cos \theta_{s}\right), & \text { reflection } \\
\left(\sin \theta_{s},-\cos \theta_{s}\right), & \text { transmission }
\end{aligned}\right.
\end{aligned}
$$

where $\theta_{i}$ and $\theta_{s}$ refer to the incidence and scattering angles with respect to the normal of the surface mean plane.

The rms value $\sigma$ of $h\left(x_{1}\right)$ is normally used as a measure of the height scale of the surface. Besides, the lateral variation of $h\left(x_{1}\right)$ is characterized by the correlation function, and it is assumed to be a Gaussian in this article Ogilvy (1991):

$$
W\left(\Delta x_{1}\right)=\frac{<h\left(x_{1}\right) h\left(x_{1}+\Delta x_{1}\right)>}{\sigma^{2}}=\exp \left(-\frac{\Delta x_{1}^{2}}{\lambda_{0}^{2}}\right)
$$

where $\lambda_{0}$ is called the correlation length, as the distance over which the correlation function falls by $1 / e$.

\subsection{Kirchhoff theory}

We assume linear, isotropic and homogeneous elastic media as described in textbooks in continuum mechanics Landau and Lifshitz (1970); Graff (1975); Achenbach (2003) amongst many others, and draw in particular on Achenbach (2003). The elastodynamic KA is illustrated in Fig. 2 at one local surface point, insonified by a $\mathrm{P}$ wave. The KA assumes that the motion of this local point is 
the same as if it were part of an infinite tangential plane insonified by the incident wave Ogilvy (1991). Physically this has an implicit limitation to having the correlation length greater than the wavelength and furthermore, in this limit, the excitation of Rayleigh surface waves will not be relevant, as an incident plane wave on a flat surface does not generate Rayleigh waves. In calculations where the correlation length is allowed to vary Maznev (2015), the Rayleigh wave contribution only becomes noticeable for small correlation lengths.

Following the continuity of displacement and traction on the interface, the total surface displacement and traction are expressed as the summation of the incident and reflection fields, which also equals those of the transmission field:

$$
\begin{aligned}
& \boldsymbol{u}=\boldsymbol{d}_{0}+R_{p p} \boldsymbol{d}_{p}^{(1)}+R_{p s} \boldsymbol{d}_{s}^{(1)}=D_{p p} \boldsymbol{d}_{p}^{(2)}+D_{p s} \boldsymbol{d}_{s}^{(2)} \\
& \boldsymbol{t}=\boldsymbol{t}_{0}+\boldsymbol{t}_{p}^{(1)}+\boldsymbol{t}_{s}^{(1)}=\boldsymbol{t}_{p}^{(2)}+\boldsymbol{t}_{s}^{(2)} .
\end{aligned}
$$

Where $\boldsymbol{u}$ and $\boldsymbol{t}$ are the displacements and tractions respectively and $R_{p p}, R_{p s}$, $D_{p p}$ and $D_{p s}$ are the Fresnel reflection and transmission coefficients of $\mathrm{P}$ and mode converted S waves respectively Achenbach (2003). The $\boldsymbol{d}_{0}, \boldsymbol{d}_{p}^{(1)}, \boldsymbol{d}_{s}^{(1)}, \boldsymbol{d}_{p}^{(2)}$ and $\boldsymbol{d}_{s}^{(2)}$ are the displacement polarization vectors for the incident $\mathrm{P}$, reflected and transmitted $\mathrm{P} / \mathrm{S}$ waves, as shown in Fig. 2, and the corresponding tractions from incident and reflected/transmitted waves are denoted as $\boldsymbol{t}_{0}, \boldsymbol{t}_{p}^{(1)}, \boldsymbol{t}_{s}^{(1)}, \boldsymbol{t}_{p}^{(2)}$ and $\boldsymbol{t}_{s}^{(2)}$. These values of tractions are obtained from the displacement field according to Hooke's law and the stress-strain relationship. In Fig. 2, the local incident/scattering angles with respect to the normal of the tangential plane are denoted as $\alpha_{0}, \alpha_{p p}, \alpha_{p s}, \beta_{p p}$ and $\beta_{p s}$, and they obey the Snell's law.

By moving $\boldsymbol{d}_{0}$ and $\boldsymbol{t}_{0}$ to one side of Eq. (4) respectively, Eq. (4) is conveniently recast as a matrix equation:

$$
\begin{aligned}
& \mathbf{M} \boldsymbol{X}=\boldsymbol{b} \\
& \boldsymbol{X}=\left[\begin{array}{llll}
R_{p p} & R_{p s} & D_{p p} & D_{p s}
\end{array}\right]^{T}
\end{aligned}
$$

where $\mathbf{M}$ is a $4 \times 4$ matrix, and $\boldsymbol{b}$ is a $4 \times 1$ vector representing the incident field. For the case with an incident $\mathrm{P}$ wave, the expressions of $\mathbf{M}$ and $\boldsymbol{b}$ are:

$$
\mathbf{M}=\left[\begin{array}{cccc}
-\cos \alpha_{p p} & \sin \alpha_{p s} & -\cos \beta_{p p} & \sin \beta_{p s} \\
-\sin \alpha_{p p} & -\cos \alpha_{p s} & \sin \beta_{p p} & \cos \beta_{p s} \\
-\cos 2 \alpha_{p s} & \frac{c_{s}^{(1)}}{c_{p}^{(1)}} \sin 2 \alpha_{p s} & \frac{\rho^{(2)}}{\rho^{(1)}} \frac{c_{p}^{(2)}}{c_{p}^{(1)}} \cos 2 \beta_{p s} & -\frac{\rho^{(2)}}{\rho^{(1)}} \frac{c_{s}^{(2)}}{c_{p}^{(1)}} \sin 2 \beta_{p s} \\
-\sin 2 \alpha_{p p} & \frac{c_{p}^{(1)}}{c_{s}^{(1)}} \cos 2 \alpha_{p s} & \frac{\rho^{(2)}}{\rho^{(1)}} \frac{c_{p}^{(1)}}{c_{p}^{(2)}}\left(\frac{c_{s}^{(2)}}{c_{s}^{(1)}}\right)^{2} \sin 2 \beta_{p p} & -\frac{\rho^{(2)}}{\rho^{(1)}} \frac{c_{s}^{(2)}}{c_{s}^{(1)}} \frac{c_{p}^{(1)}}{c_{s}^{(1)}} \cos 2 \beta_{p s}
\end{array}\right]
$$

and

$$
\boldsymbol{b}=\left[\begin{array}{llll}
-\cos \alpha_{0} & \sin \alpha_{0} & \cos 2 \alpha_{p s} & -\sin 2 \alpha_{0}
\end{array}\right]^{T}
$$

where $\rho^{(1)}$ and $\rho^{(2)}$ refer to the density of Solid 1 and Solid 2. $c_{p}^{(1)}, c_{s}^{(1)}, c_{p}^{(2)}$ and $c_{s}^{(2)}$ are compressional or shear wave speed in Solid 1 or 2. By solving for $\boldsymbol{X}$ in 

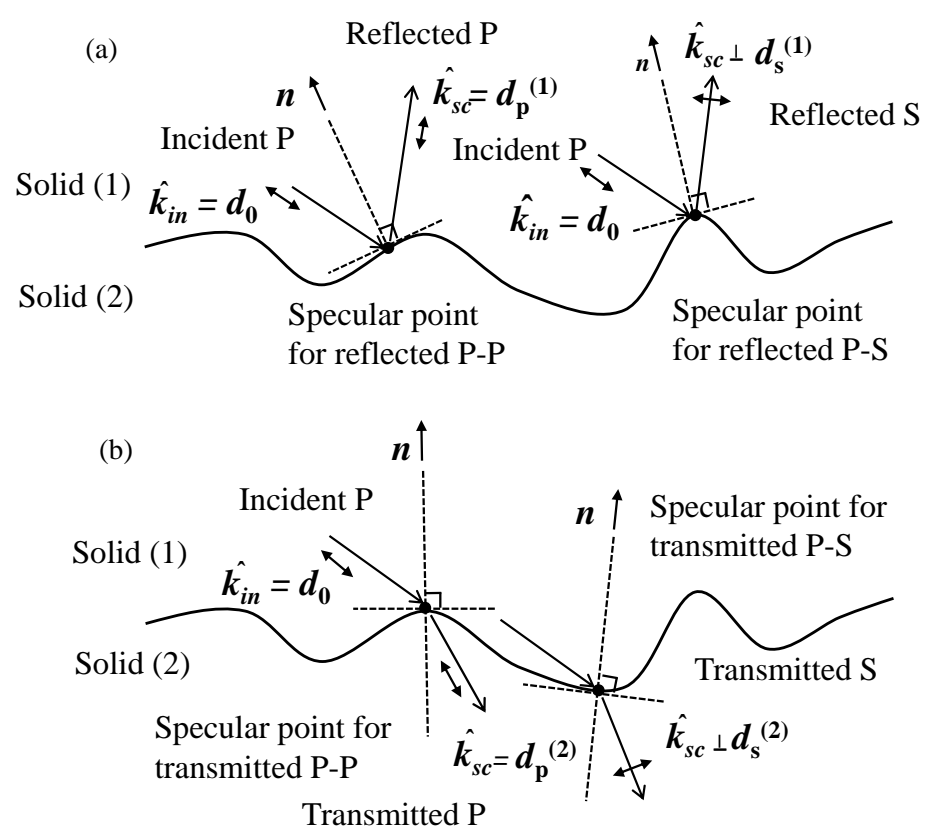

Figure 3: Specular points for different modes. (a) Reflection P-P and P-S modes. (b) Transmission P-P and P-S modes

the linear system of Eq. (5), one obtains the Fresnel reflection/transmission coefficients, which are position-dependent along the interface; the total displacement $\boldsymbol{u}$ and subsequently the traction $\boldsymbol{t}$ at one surface point can then be calculated by substituting the solved Fresnel coefficients into Eq. (4).

The elastodynamic Helmholtz integral formula Achenbach (2003) with the integral taken along the rough interface $\mathcal{S}$ :

$$
\begin{array}{r}
u_{k}(\boldsymbol{r})=\int_{\mathcal{S}}\left[\sigma_{i j ; k}^{G}\left(\left|\boldsymbol{r}-\boldsymbol{r}_{0}\right|\right) u_{i}\left(\boldsymbol{r}_{0}\right) n_{j}\left(\boldsymbol{r}_{0}\right)\right. \\
\left.-\sigma_{i j}\left(\boldsymbol{r}_{0}\right) u_{i ; k}^{G}\left(\left|\boldsymbol{r}-\boldsymbol{r}_{0}\right|\right) n_{j}\left(\boldsymbol{r}_{0}\right)\right] d S\left(\boldsymbol{r}_{0}\right) \\
\text { where } i, j, k=1,2
\end{array}
$$

is key and is used to calculate the scattered displacement $u_{k}(\boldsymbol{r})$ where $\boldsymbol{r}$ is the vector indicating the position of the observation point, and $n_{j}$ is the unit normal vector pointing outside the surface. In Eq. (8) $u_{i}\left(\boldsymbol{r}_{0}\right)$ and $\sigma_{i j}\left(\boldsymbol{r}_{0}\right)$ represent the displacement and stress at a point $\boldsymbol{r}_{0}$ on the interface; also required are the elastodynamic Green's functions in the unbounded domain $u_{i ; k}^{G}\left(\left|\boldsymbol{r}-\boldsymbol{r}_{0}\right|\right)$ and $\sigma_{i j ; k}^{G}\left(\left|\boldsymbol{r}-\boldsymbol{r}_{0}\right|\right)$. The Green's function can be expressed explicitly as Achenbach 
(2003):

$$
\begin{aligned}
u_{i ; k}^{G}\left(\left|\boldsymbol{r}-\boldsymbol{r}_{0}\right|\right) & =\frac{1}{\mu k_{s}^{2}}\left[-G\left(k_{p}\left|\boldsymbol{r}-\boldsymbol{r}_{0}\right|\right)+G\left(k_{s}\left|\boldsymbol{r}-\boldsymbol{r}_{0}\right|\right)\right]_{, i k} \\
& +G\left(k_{s}\left|\boldsymbol{r}-\boldsymbol{r}_{0}\right|\right) \delta_{i k} \\
\sigma_{i j ; k}^{G}\left(\left|\boldsymbol{r}-\boldsymbol{r}_{0}\right|\right) & =\left(1-2 k_{p}^{2} / k_{s}^{2}\right) G\left(k_{p}\left|\boldsymbol{r}-\boldsymbol{r}_{0}\right|\right)_{, k} \delta_{i j} \\
& -\frac{2}{k_{s}^{2}}\left[G\left(k_{p}\left|\boldsymbol{r}-\boldsymbol{r}_{0}\right|\right)-G\left(k_{s}\left|\boldsymbol{r}-\boldsymbol{r}_{0}\right|\right)\right]_{, i j k} \\
& +G\left(k_{s}\left|\boldsymbol{r}-\boldsymbol{r}_{0}\right|\right)_{, j} \delta_{i k}+G\left(k_{s}\left|\boldsymbol{r}-\boldsymbol{r}_{0}\right|\right)_{, i} \delta_{j k}
\end{aligned}
$$

where $k_{p}$ and $k_{s}$ are the compressional and shear wavenumber, and $\mu$ is the Lamé second parameter. The notation $f_{, i}=\frac{\partial f}{\partial x_{i}}$ is used here, and $\delta$ refers to the Dirac delta function. The function $G\left(k_{\beta}\left|\boldsymbol{r}-\boldsymbol{r}_{0}\right|\right)$ is the acoustic Green's function, which in $2 \mathrm{D}$ is expressed as:

$$
G\left(k_{\beta}\left|\boldsymbol{r}-\boldsymbol{r}_{0}\right|\right)=-\frac{i}{4} H_{0}^{(1)}\left(k_{\beta}\left|\boldsymbol{r}-\boldsymbol{r}_{0}\right|\right)
$$

Here $H_{0}^{(1)}\left(k_{\beta}\left|\boldsymbol{r}-\boldsymbol{r}_{0}\right|\right)$ denotes the zero order Hankel function of the first kind.

Our interest is in the far field and so we take the far field approximation that $\left|\boldsymbol{r}-\boldsymbol{r}_{\mathbf{0}}\right| \approx r-\hat{\boldsymbol{r}} \cdot \boldsymbol{r}_{\mathbf{0}}$, and the Hankel function can be approximated as:

$$
H_{0}^{(1)}\left(k_{\beta}\left|\boldsymbol{r}-\boldsymbol{r}_{0}\right|\right) \approx\left(\frac{2}{\pi k_{\beta}}\right)^{1 / 2} e^{-i \pi / 4}\left(e^{i k_{\beta} r} / \sqrt{r}\right) e^{-i k_{\beta} \hat{\boldsymbol{r}} \cdot \boldsymbol{r}_{\mathbf{O}}}
$$

In $2 \mathrm{D} \mathrm{Eq.} 8$ is then transferred into an integral along the mean plane of the surface $\mathcal{S}_{m}$ :

$$
\begin{array}{r}
\boldsymbol{u}_{\beta}(\boldsymbol{r})=-i k_{\beta} \sqrt{\frac{2 \pi i}{k_{\beta}}} \frac{\exp \left(i k_{\beta} r\right)}{4 \pi \sqrt{r}} \int_{\mathcal{S}_{m}}\left(\boldsymbol{U}_{\beta}+\frac{\boldsymbol{T}_{\beta}}{i k_{\beta} \rho c_{\beta}^{2}}\right) \\
\exp \left(i k_{\beta} \phi_{\beta}\right) d \mathcal{S}_{m} \quad \beta=p, s
\end{array}
$$

where $k_{\beta}$ and $c_{\beta}$ refer to the wavenumber and wavespeed of the scattered waves, and $\rho$ is the density. They are different for Solid 1(reflection) and Solid 2(transmission). The phase term $\phi_{\beta}=A_{\beta} x_{1}+C_{\beta} h\left(x_{1}\right)$, and

$$
\begin{aligned}
& A_{\beta}= \frac{k_{p}^{(1)}}{k_{\beta}} \sin \theta_{i}-\sin \theta_{s} \\
& C_{\beta}= \begin{cases}-\left(\frac{k_{p}^{(1)}}{k_{\beta}^{(1)}} \cos \theta_{i}+\cos \theta_{s}\right), & \text { reflection } \\
-\left(\frac{k_{p}^{(1)}}{k_{\beta}^{(2)}} \cos \theta_{i}-\cos \theta_{s}\right), & \text { transmission }\end{cases}
\end{aligned}
$$

where $k_{p}^{(1)}$ stands for the wavenumber of the incident compressional waves from Solid 1. We emphasise that $C_{\beta}$ has different expressions for reflection and 
transmission. The terms $\boldsymbol{U}_{\beta}$ and $\boldsymbol{T}_{\beta}$ represent the decomposed boundary displacement and traction for different wave modes and they are expressed as:

$$
\begin{aligned}
\boldsymbol{U}_{p} & =\left[(\boldsymbol{u} \cdot \boldsymbol{N})\left(1-2\left(\frac{c_{s}}{c_{p}}\right)^{2}\right)+2\left(\frac{c_{s}}{c_{p}}\right)^{2}\left(\boldsymbol{u} \cdot \hat{\boldsymbol{k}}_{\boldsymbol{s c}}\right)\left(\boldsymbol{N} \cdot \hat{\boldsymbol{k}}_{\boldsymbol{s c}}\right)\right] \hat{\boldsymbol{k}}_{\boldsymbol{s c}} \\
\boldsymbol{U}_{s} & =\left(\boldsymbol{N} \cdot \hat{\boldsymbol{k}}_{\boldsymbol{s c}}\right) \boldsymbol{u}+\left(\boldsymbol{u} \cdot \hat{\boldsymbol{k}}_{\boldsymbol{s c}}\right) \boldsymbol{N}-2\left(\boldsymbol{u} \cdot \hat{\boldsymbol{k}}_{\boldsymbol{s c}}\right)\left(\boldsymbol{N} \cdot \hat{\boldsymbol{k}}_{\boldsymbol{s c}}\right) \hat{\boldsymbol{k}}_{\boldsymbol{s c}} \\
\boldsymbol{T}_{p} & =\left(\hat{\boldsymbol{k}}_{\boldsymbol{s c}} \cdot \boldsymbol{t}\right) \hat{\boldsymbol{k}}_{\boldsymbol{s c}} \\
\boldsymbol{T}_{s} & =\boldsymbol{t}-\left(\hat{\boldsymbol{k}}_{\boldsymbol{s c}} \cdot \boldsymbol{t}\right) \hat{\boldsymbol{k}}_{\boldsymbol{s c}}
\end{aligned}
$$

in which $\boldsymbol{N}$ is the unnormalized vector normal to the surface, and it equals $\left(-\partial h / \partial x_{1}, 1\right)$. The interface displacement $\boldsymbol{u}$ and traction $\boldsymbol{t}$ are obtained using the KA from Eq. (4) - (7). We assume an incident $\mathrm{P}$ wave in this article, and hence neglect the subscript $\alpha$ denoting the incident mode. Note that the same methodology can be equivalently applied to an incident $\mathrm{S}$ wave. For shear wave incidence, one needs to revise Eq. (4) - (7) to calculate the Fresnel reflection/transmission coefficients representing a plane $\mathrm{S}$ wave reflected from the tangential plane.

It is necessary to remove the surface slope from the integration in Eq. 12, for the analytical derivation of the ensemble averaging $<u_{k} \bar{u}_{j}>$ later (the overline denoting the complex conjugate). Following the method described in Shi et al. (2016), we apply a stationary phase approach to Eq. (12) to approximate $\boldsymbol{U}_{\beta}$ and $\boldsymbol{T}_{\beta}$. The first order derivatives of the phase term $\phi_{\beta}$ with respect to $x_{1}$ is set to be zero to locate the stationary phase points, and the following expression is obtained:

$$
\frac{\partial h}{\partial x_{1}}=-\frac{A_{\beta}}{C_{\beta}} .
$$

The underlying physics of this is that the varying slope along the surface is approximated as constant for given incidence/scattering angles, wave modes and material properties; the stationary phase points are also called 'specular points' in the optical community Berry (2015). At these points, the scattering direction is viewed the same as the specular direction (reflection or transmission) to the incidence angle with respect to the local normal vector, as illustrated in Fig. 3 165 for both the reflection and transmission modes. The boundary integral Eq. (12) is therefore dominated by contributions from these points where the phase term $\phi_{\beta}$ is stationary.

By substituting the slope approximation in Eq. 15) into Eq. 12 - 14, the values of $\boldsymbol{U}_{\beta}$ and $\boldsymbol{T}_{\beta}$ at the 'specular points' are calculated. Here we assume that

$$
\boldsymbol{F}_{\beta}=1 / 2\left(\boldsymbol{U}_{\beta}+\frac{\boldsymbol{T}_{\beta}}{i k_{\beta} \rho c_{\beta}^{2}}\right),
$$

which is called the elastodynamic angular factor hereinafter. The function $\boldsymbol{F}_{\beta}$ depends on the incidence/scattering angle, material properties in both media 
and wave modes, and it is a vector containing two components corresponding to the polarization of the displacement. By eliminating the dependence of the surface gradient, Eq. (12) is simplified as:

$$
\boldsymbol{u}_{\beta}(\boldsymbol{R})=-i k_{\beta} \sqrt{\frac{2 \pi i}{k_{\beta}}} \frac{\exp \left(i k_{\beta} r\right)}{4 \pi \sqrt{r}} 2 \boldsymbol{F}_{\boldsymbol{\beta}} \int_{\mathcal{S}_{m}} \exp \left(i k_{\beta} \phi_{\beta}\right) d \mathcal{S}
$$

170

\subsection{Expected scattering cross section}

We are now in a position to derive the expression for the scattering cross section, an important normalized metric commonly used. The expected scattering cross section $\left(\left\langle\sigma_{s c, \beta}\right\rangle\right)$ for waves scattered from a rough surface is expressed as Achenbach (2003):

$$
<\sigma_{s c, \beta}>=\frac{r<\boldsymbol{P}_{s c, \beta} \cdot \hat{\boldsymbol{k}}_{\boldsymbol{s c}}>}{E_{\text {inc }}}
$$

where $\left\langle\boldsymbol{P}_{s c, \beta} \cdot \hat{\boldsymbol{k}}_{\boldsymbol{s c}}\right\rangle$ is the scattering power flux per unit area of the plane perpendicular to the scattering vector. $E_{\text {inc }}$ refers to the incident energy which equals the incident power flux $\boldsymbol{P}_{i n c}$ integrated over the surface mean plane, and it is expressed as:

$$
E_{i n c}=\int_{-\infty}^{\infty} \boldsymbol{P}_{i n c} \cdot \hat{\boldsymbol{x}}_{\mathbf{2}} d x_{1}
$$

where $\hat{\boldsymbol{x}}_{\mathbf{2}}$ denotes the unit vector in the $x_{2}$ direction normal to the surface. The $i$ th component of the power flux $P$ is defined as:

$$
\begin{aligned}
& <P_{i}>=<\tau_{i j} \frac{\partial u_{j}}{\partial t}>= \\
& -i \omega\left[\lambda i k_{q}<u_{q} \bar{u}_{j}>\delta_{i j}+\mu\left(i k_{j}<u_{i} \bar{u}_{j}>+i k_{i}<u_{j} \bar{u}_{j}>\right)\right]
\end{aligned}
$$

where $\tau_{i j}$ is the stress, $k_{i}$ is the $i$ th component of the wavevector, $u_{i}$ is the displacement and $\bar{u}_{i}$ is its conjugate value. $\lambda$ and $\mu$ are Lamé first and second parameters, which are different for Solid 1 and Solid 2.

The displacement of the incident wave is assumed to be a Gaussian tapered plane wave with the form given as Schultz and Tokosz (1994):

$$
\begin{aligned}
\boldsymbol{\Psi}\left(x_{1}, x_{2}\right) & =\boldsymbol{d}_{0} \exp \left\{i k_{p}^{(1)}\left(x_{1} \sin \theta_{0}-x_{2} \cos \theta_{0}\right)\left[1+\eta\left(x_{1}, x_{2}\right)\right]\right. \\
& \left.\left.-\left[\left(x_{1} \cos \theta_{0}+x_{2} \sin \theta_{0}\right) / w\right]^{2}\right]\right\} \\
\eta\left(x_{1}, x_{2}\right) & =\frac{c_{p}^{(1) 2}}{\omega^{2} w^{2}}\left[\frac{2}{w^{2}}\left(x_{1} \cos \theta_{0}+x_{2} \sin \theta_{0}\right)^{2}-1\right]
\end{aligned}
$$

where $w$ is the half-width of the Gaussian beam, at which the value of the incident amplitude falls by $1 / e . c_{p}^{(1)}$ and $k_{p}^{(1)}$ are the compressional wavespeed and wavenumber in Solid 1. By substituting Eq. (20) into Eqs. 19 18), the 
incident energy $E_{\text {inc }}$ through the mean surface plane has been derived by Schultz and Tokosz (1994):

$$
\begin{aligned}
E_{\text {inc }} & =\frac{\sqrt{\pi} w \omega^{2}}{\sqrt{2} c_{p}^{(1)}} P_{B} \\
P_{B} & =\left[1-\frac{c_{p}^{(1) 2}}{\omega^{2} w^{2}}\left(1+2 \tan ^{2} \theta_{0}\right)\right]\left[\left(\lambda^{(1)}+2 \mu^{(1)}\right) \cos ^{2} \theta_{0}\right. \\
& \left.+\mu^{(1)} \sin ^{2} \theta_{0}\right] \\
& \left.+\left[\left(1+\frac{1}{2} \frac{c_{p}^{(1) 2}}{w^{2} \omega^{2}}\right) \tan \theta_{0}\right)\right]\left(\lambda^{(1)}+\mu^{(1)}\right) \sin \theta_{0} \cos \theta_{0}
\end{aligned}
$$

From Eqs. (17) to (19), the calculation of the ensemble averaging $\left\langle\sigma_{s c, \beta}\right\rangle$ is reduced to deriving the expression for $\left\langle u_{i} \bar{u}_{j}\right\rangle$. According to Eq. (16), $<u_{i} \bar{u}_{j}>$ of the scattered waves is:

$$
\begin{aligned}
<u_{i} \bar{u}_{j}> & =\frac{k_{\beta} F_{i, \beta} F_{j, \beta}}{2 \pi r} \int_{\mathcal{S}} \int_{\mathcal{S}} \Psi\left(x_{1}\right) \Psi\left(x_{1}^{\prime}\right) e^{i k_{\beta} A_{\beta}\left(x_{1}-x_{1}^{\prime}\right)} \\
& <e^{i k_{\beta} C_{\beta}\left(h-h^{\prime}\right)}>d x_{1} d x_{1}^{\prime}
\end{aligned}
$$

where the function $\Psi\left(x_{1}\right)$ represents the incident displacement field along the mean surface calculated from the Gaussian tapered plane wave in Eq. (20). Following the derivation in Shi et al. (2016), Eq. (22) is simplified via a change of variable that $\Delta x_{1}=x_{1}^{\prime}-x_{1}$

$$
\begin{gathered}
<u_{i} \bar{u}_{j}>=\frac{k_{\beta} F_{i, \beta} F_{j, \beta}}{2 \pi r} \int_{-\infty}^{\infty} \int_{-\infty}^{\infty} \Psi\left(x_{1}\right) \Psi\left(\Delta x_{1}+x_{1}\right) d x_{1} \\
e^{i k_{\beta} A_{\beta} \Delta x_{1}} \chi_{2}\left(k_{\beta} C_{\beta}, \Delta x_{1}\right) d \Delta x_{1}
\end{gathered}
$$

where $\chi_{2}\left(k_{\beta} C_{\beta}, \Delta x_{1}\right)$ is called the two-dimensional characteristic function Ogilvy (1991). If the surface height follows the Gaussian distribution, $\chi_{2}\left(k_{\beta} C_{\beta}, \Delta x_{1}\right)$ then has an analytical form:

$$
\chi_{2}\left(k_{\beta} C_{\beta}, \Delta x_{1}\right)=\exp \left[-g_{\beta}\left(1-W\left(\Delta x_{1}\right)\right)\right]
$$

where the parameter $g_{\beta}$ is different from Eq. (1), as it includes both reflection and transmission, and non-specular angles:

$$
g_{\beta}=k_{\beta}^{2} C_{\beta}^{2} \sigma^{2}= \begin{cases}\left(k_{p}^{(1)} \cos \theta_{i}+k_{\beta}^{(1)} \cos \theta_{s}\right)^{2} \sigma^{2}, & \text { reflection } \\ \left(k_{p}^{(1)} \cos \theta_{i}-k_{\beta}^{(2)} \cos \theta_{s}\right)^{2} \sigma^{2}, & \text { transmission }\end{cases}
$$

Now assuming the first integral of $\Psi\left(x_{1}\right) \Psi\left(\Delta x_{1}+x_{1}\right)$ is a slowly varying function around the point $\Delta x_{1}=0$ as compared with $\chi_{2}\left(k_{\beta} C_{\beta}, \Delta x_{1}\right)$; this is accurate provided the half-width of the beam $w$ is larger than $3 \lambda_{p}$. Eq. (23) is 
then further simplified as:

$$
\begin{aligned}
<u_{i} \bar{u}_{j}> & \approx \frac{k_{\beta} F_{i, \beta} F_{j, \beta}}{2 \pi r} \int_{-\infty}^{\infty} \Psi\left(x_{1}\right)^{2} d x_{1} \int_{-\infty}^{\infty} e^{i k_{\beta} A_{\beta} \Delta x_{1}} \\
& \chi_{2}\left(k_{\beta} C_{\beta}, \Delta x_{1}\right) d \Delta x_{1} \\
& =\frac{k_{\beta} F_{i, \beta} F_{j, \beta}}{2 \pi r} L_{e f f} \Sigma_{n=0}^{\infty} e^{-g_{\beta}} \frac{g_{\beta}^{n}}{n !} \int_{-\infty}^{\infty} e^{i k_{\beta} A_{\beta} \Delta x_{1}} \\
& W^{n}\left(\Delta x_{1}\right) d \Delta x_{1} .
\end{aligned}
$$

Here the parameter $L_{\text {eff }}=\int_{-\infty}^{\infty} \Psi^{2}\left(x_{1}\right) d x_{1}$, is the effective insonified length. We now identify the coherent and diffuse contributions: the first term in Eq. (26) when $n=0$ corresponds to the coherent component, while the diffuse field contribution comes from higher-order terms. Substituting the Gaussian correlation function Eq. (3) into Eq. (26), the diffuse components of $\left\langle u_{i} \bar{u}_{j}\right\rangle$ are expressed as:

$$
\begin{aligned}
<u_{i} \bar{u}_{j}>^{d} & =\frac{k_{\beta} F_{i, \beta} F_{j, \beta} \lambda_{0} \sqrt{\pi} e^{-g_{\beta}}}{2 \pi r} L_{e f f} \Sigma_{n=1}^{\infty} \frac{g_{\beta}^{n}}{n ! \sqrt{n}} \\
& \exp \left[-\frac{k_{\beta}^{2} A_{\beta}^{2} \lambda_{0}^{2}}{4 n}\right]
\end{aligned}
$$

In the low frequency limit or when the rms value is very small compared with the wavelength, the scattering cross section is approximated by only keeping the first term of the Taylor series in Eq. 27) Ogilvy (1991):

$$
\begin{aligned}
<u_{i} \bar{u}_{j}>^{d} & \approx \frac{k_{\beta} F_{i, \beta} F_{j, \beta} \lambda_{0} \sqrt{\pi} e^{-g_{\beta}}}{2 \pi r} L_{e f f} g_{\beta} \exp \left[-\frac{k_{\beta}^{2} A_{\beta}^{2} \lambda_{0}^{2}}{4}\right] \\
& =\frac{k_{\beta}^{3} F_{i, \beta} F_{j, \beta} e^{-g_{\beta}}}{r} C_{\beta}^{2} L_{e f f} P\left(k_{\beta} A_{\beta}\right)
\end{aligned}
$$

where $P\left(k_{\beta} A_{\beta}\right)$ is called the power spectrum of the surface height Ogilvy (1991).

In contrast to the low frequency approximation, in the high frequency limit when $k_{\beta} \rightarrow \infty$, we assume that $k_{\beta} C_{\beta}\left(h-h^{\prime}\right) \approx k_{\beta} C_{\beta} \frac{\partial h}{\partial x_{1}}$, and hence the ensemble averaging $\left.<u_{i} \bar{u}_{j}\right\rangle$ is derived as:

$$
\begin{aligned}
<u_{i} \bar{u}_{j}>^{d} & \approx \frac{k_{\beta} F_{i, \beta} F_{j, \beta}}{2 \pi r} L_{e f f} \\
& \int_{-\infty}^{\infty}<e^{i k_{\beta}\left[\left(A_{\beta}+C_{\beta} \frac{\partial h}{\partial x}\right) \Delta x_{1}\right]}>d \Delta x_{1} \\
& =\frac{k_{\beta} F_{i, \beta} F_{j, \beta}}{2 \pi r} L_{e f f} \times \frac{2 \pi}{k_{\beta} C_{\beta}}<\delta\left(\frac{\partial h}{\partial x}+\frac{A_{\beta}}{C_{\beta}},\right)> \\
& =\frac{F_{i, \beta} F_{j, \beta}}{r C_{\beta}} L_{e f f} \int_{-\infty}^{\infty} p_{g}\left(\gamma_{x}\right) d \gamma_{x} \delta\left(\gamma_{x}+\frac{A_{\beta}}{C_{\beta}}\right) \\
& =\frac{F_{i, \beta} F_{j, \beta}}{r C_{\beta}} L_{e f f} \times p_{g}\left(\frac{\partial h}{\partial x}=-\frac{A_{\beta}}{C_{\beta}}\right)
\end{aligned}
$$




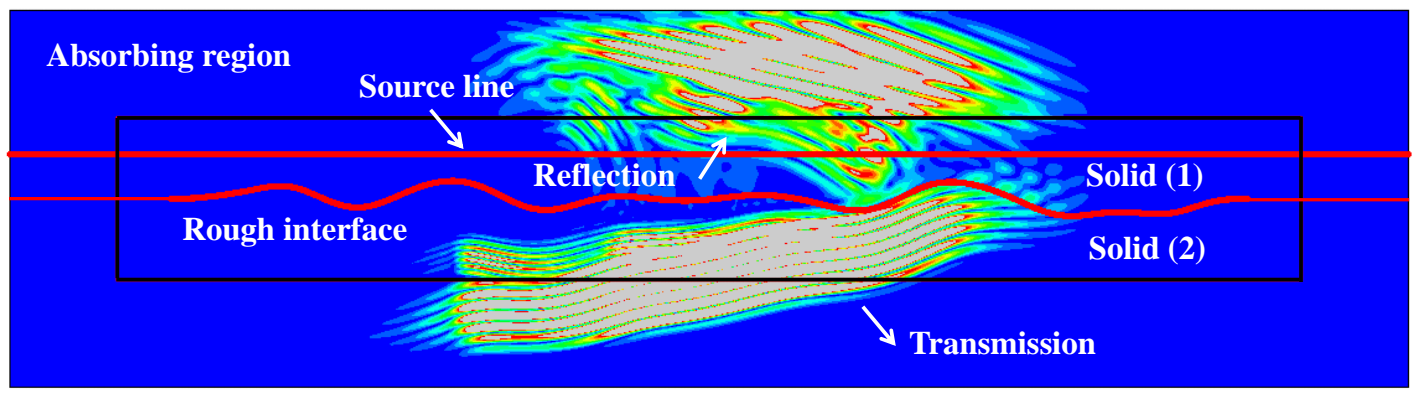

Figure 4: Snapshot of animation in the local FE box showing the waves reflected and transmitted by the rough interface. Details of the simulation are given in the text.
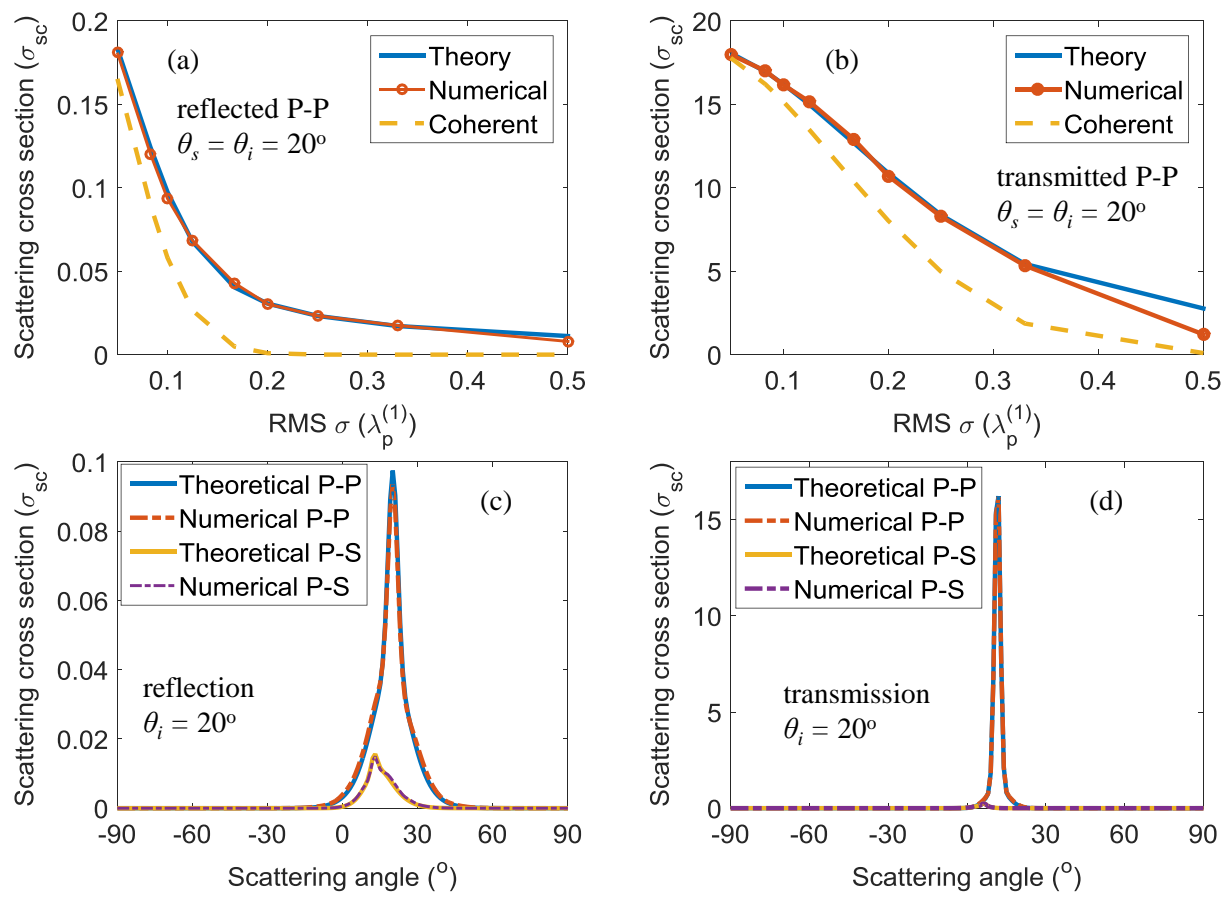

Figure 5: Comparison of the theoretical results with those from numerical simulations $\left(\theta_{i}=\right.$ $20^{\circ}$; Solid 1: Si, Solid 2:Ge). (a) Specular $\sigma_{s c}$ of the reflected P-P mode. (b) Specular $\sigma_{s c}$ of the transmitted P-P mode. (c) Angular distribution of $\sigma_{s c}$ of the reflected P-P and P-S modes when $\sigma=\lambda_{p}^{(1)} / 8, \lambda_{0}=2 \lambda_{p}^{(1)}$. (d) Angular distribution of $\sigma_{s c}$ of the transmitted P-P and P-S modes when $\sigma=\lambda_{p}^{(1)} / 8, \lambda_{0}=2 \lambda_{p}^{(1)}$. 
where $p_{g}$ is the probability function of the surface slope, defined as:

$$
p_{g}\left(\frac{\partial h}{\partial x_{1}}\right)=\frac{\lambda_{0}}{2 \sqrt{\pi} \sigma} \exp \left[-\frac{\left(\frac{\partial h}{\partial x_{1}}\right)^{2} \lambda_{0}^{2}}{4 \sigma^{2}}\right]
$$
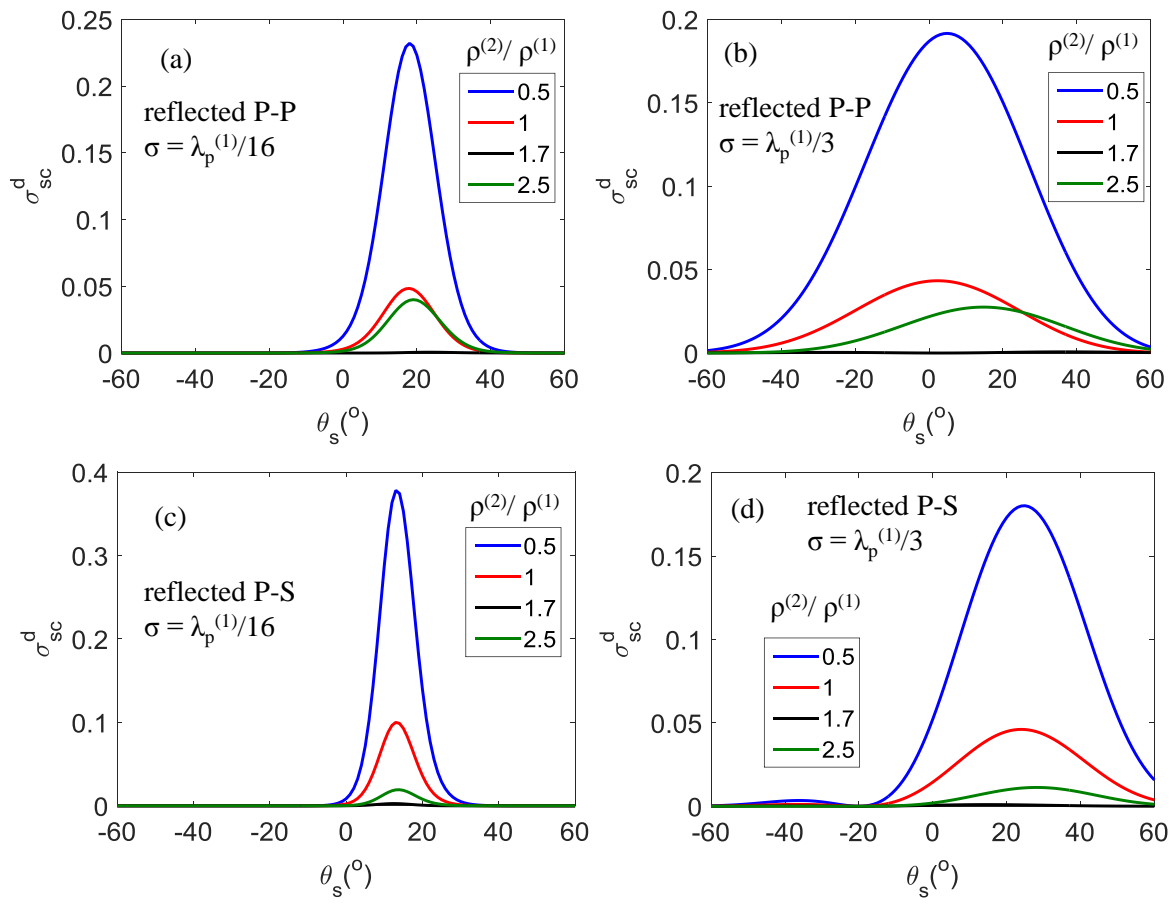

Figure 6: Diffuse scattering cross section $\left(\sigma_{s c}^{d}\right.$, reflection) from rough interfaces (Solid 1 is $\mathrm{Si}$; Solid 2 has the same wave speed as Ge but various values of the density.) by a $20^{\circ}$ incident $\mathrm{P}$ wave as a function of scattering angles. (a) Reflected P-P mode, $\sigma=\lambda_{p}^{(1)} / 16$. (b) Reflected P-P mode, $\sigma=\lambda_{p}^{(1)} / 3$. (c) Reflected P-S mode, $\sigma=\lambda_{p}^{(1)} / 16$. (d) Reflected P-S mode, $\sigma=$ $\lambda_{p}^{(1)} / 3$. The correlation length $\lambda_{0}=2 \lambda_{p}^{(1)}$.

The scattering cross section $\left\langle\sigma_{s c}\right\rangle$ is similarly decomposed into the coherent and the diffuse fields:

$$
\begin{aligned}
<\sigma_{s c}> & =\sigma_{s c}^{c}+\sigma_{s c}^{d} \\
& =\sigma_{s c}^{\text {flat }} \exp \left(-g_{\beta}\right)+\sigma_{s c}^{d}
\end{aligned}
$$

The coherent scattering cross section is a simple exponential decay in terms of 180 that from a flat surface. The diffuse scattering cross section $\sigma_{s c}^{d}$ is obtained by substituting the expression of Eq. (27) into Eq. (17) - Eq. (19) . 


\section{Numerical Verification}
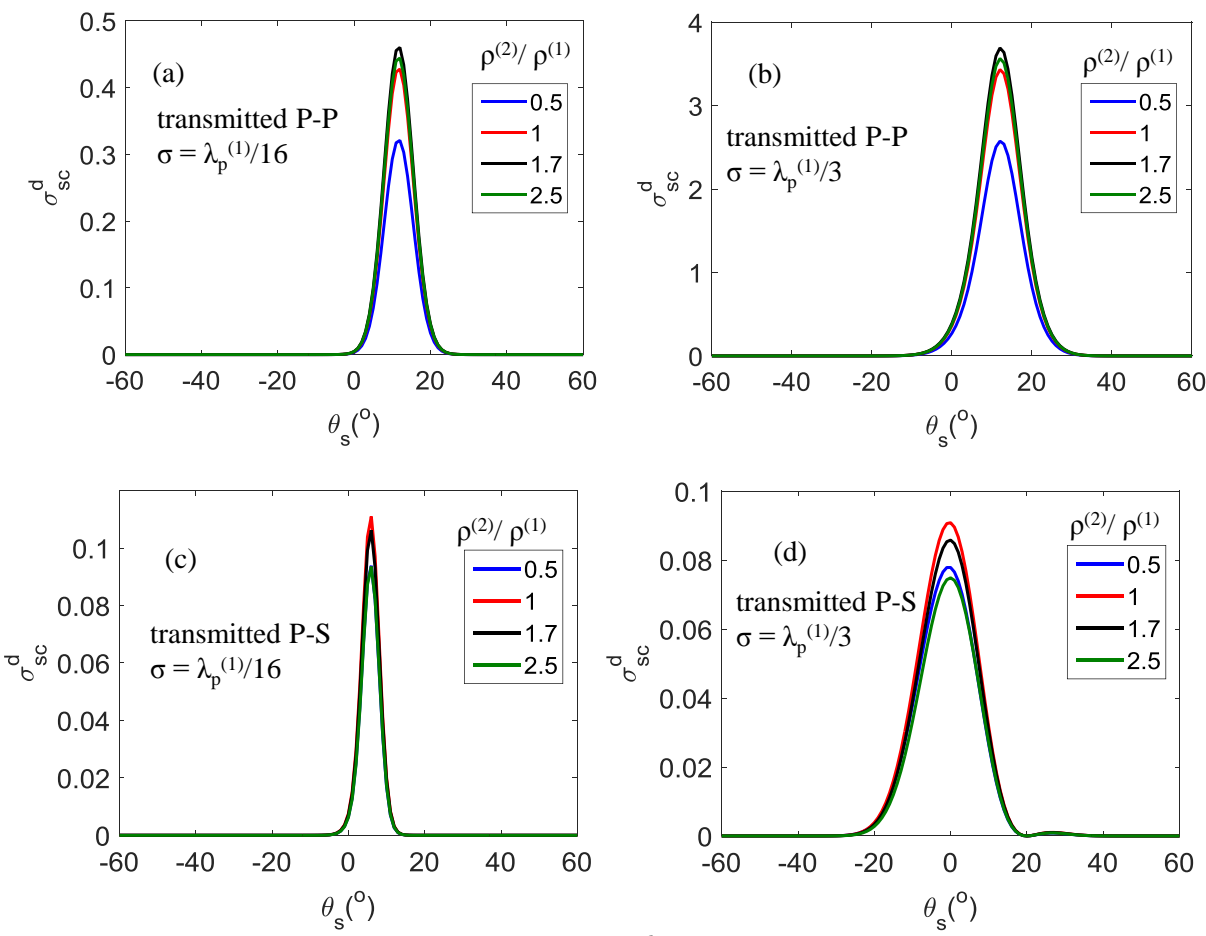

Figure 7: Diffuse scattering cross section $\left(\sigma_{s c}^{d}\right.$, transmission) from rough interfaces (Solid 1 is $\mathrm{Si}$; Solid 2 has the same wave speed as Ge but various values of the density.) by a $20^{\circ}$ incident $\mathrm{P}$ wave as a function of scattering angles $\left(\theta_{s}\right)$. (a) Transmitted P-P mode, $\sigma=\lambda_{p}^{(1)} / 16$. (b) Transmitted P-P mode, $\sigma=\lambda_{p}^{(1)} / 3$. (c) Transmitted P-S mode, $\sigma=\lambda_{p}^{(1)} / 16$. (d) Transmitted P-S mode, $\sigma=\lambda_{p}^{(1)} / 3$. The correlation length $\lambda_{0}=2 \lambda_{p}^{(1)}$.

In this section, the predictions from the formulae for $\left\langle\sigma_{s c}\right\rangle$ are compared with full numerical Monte Carlo simulations; these simulations use Gaussian surfaces from low to high roughnesses $\left(\sigma=\lambda_{p}^{(1)} / 20 \sim \lambda_{p}^{(1)} / 2, \lambda_{0}=2 \lambda_{p}^{(1)}, \lambda_{p}^{(1)}\right.$ being the compressional wavelength in Solid 1$)$. For each realization of the surface a purely numerical method, the finite element boundary integral (FEBI) approach Shi et al. (2015, 2014), is performed to compute $\sigma_{s c}$. The FEBI

190 method is highly efficient as it computes the very local scattering field on the interface using an explicit time domain FE scheme inside a small domain, and then performs a boundary integral to globally calculate the scattered waves. This approach significantly reduces the computational burden for rough surface calculations.

195

For the local FE computation, to yet further optimise the numerical simulations, we take advantage of the recently developed GPU driven FE software 
Pog Huthwaite (2014) and this greatly accelerates the simulation. The local FE model also takes advantage of developments in absorbing regions Rajagopal et al. (2012) to prevent spurious reflections from the edges of the small computational domain from contaminating the results.

Fig. 4 shows a snapshot from a typical local FE simulation of waves scattered from a rough interface. The two bulk media we take as example materials are 205 Silicon (Solid 1) and Germanium (Solid 2), as they are typical media of interest, with densities and wave speeds of $2329 \mathrm{~kg} / \mathrm{m}^{3}, 8320 \mathrm{~m} / \mathrm{s}$, and $5246 \mathrm{~m} / \mathrm{s}$ for Silicon and $5323 \mathrm{~kg} / \mathrm{m}^{3}, 4866 \mathrm{~m} / \mathrm{s}$, and $2771 \mathrm{~m} / \mathrm{s}$ for Germanium. The FE model has a dimension of $37 \lambda_{p}^{(1)} \times 10 \lambda_{p}^{(1)}$, including an absorbing region Rajagopal et al. (2012) with a thickness of $3 \lambda_{p}^{(1)}$. The rough interface separating two media has 210 a length of $29 \lambda_{p}^{(1)}$. A source line is placed $1.2 \lambda_{p}^{(1)}$ above the rough interface on the Si side. It excites a Gaussian tapered plane $\mathrm{P}$ wave with a half beam width $w$ of $5 \lambda_{p}^{(1)}$ insonifying the interface, at an incidence angle of $\theta_{i}=20^{\circ}$; the use of the Gaussian tapered incident wave eliminates the edge effects Schultz and Tokosz (1994). The incident wave is assumed to be a five-cycle tone burst.

After computing the displacement and the stress on the interface, a boundary integral along the interface is performed to calculate the scattered waves. The FEBI method is run on each realization and the sample averaged scattering cross section from all realizations is then used to approximate the ensemble averaging 220 against which we assess the accuracy of the analytical formulae. Specifically, surfaces with the same correlation length $\left(\lambda_{0}=2 \lambda_{p}^{(1)}\right)$, and rms values ranging from $\lambda_{p}^{(1)} / 20$ to $\lambda_{p}^{(1)} / 2$ are used to find the valid region of the analytical solution. For each roughness, 400 realizations of surfaces are generated Thorsos (1988) for simulations to obtain the mean scattering cross section.

225

Fig. 5(a) and (b) show the total scattering cross sections (i.e. coherent + diffuse) for both the reflected P-P mode in Solid 1 and the transmitted P-P mode in Solid 2 at the specular angles as a function of $\sigma$. Note that according to Snell's law, the specular angle is $20^{\circ}$ for the reflection and $11.5^{\circ}$ for the transmission; sample averaged numerical results, when $\sigma<\lambda_{p}^{(1)} / 2$, for both the reflection and transmission. The agreement indicates that the developed theoretical formulae using the Kirchhoff approximation is accurate for surfaces with a much wider range of roughness, as compared with the perturbation method Sun and Pipe conventional Kirchhoff formula using Eq. (1) is plotted, and it is always below the numerical benchmark curve due to the lack of the diffuse component. It can also be observed that the transmission cross section attenuates with increasing roughness much more slowly than that for the reflection in the specular direction. 

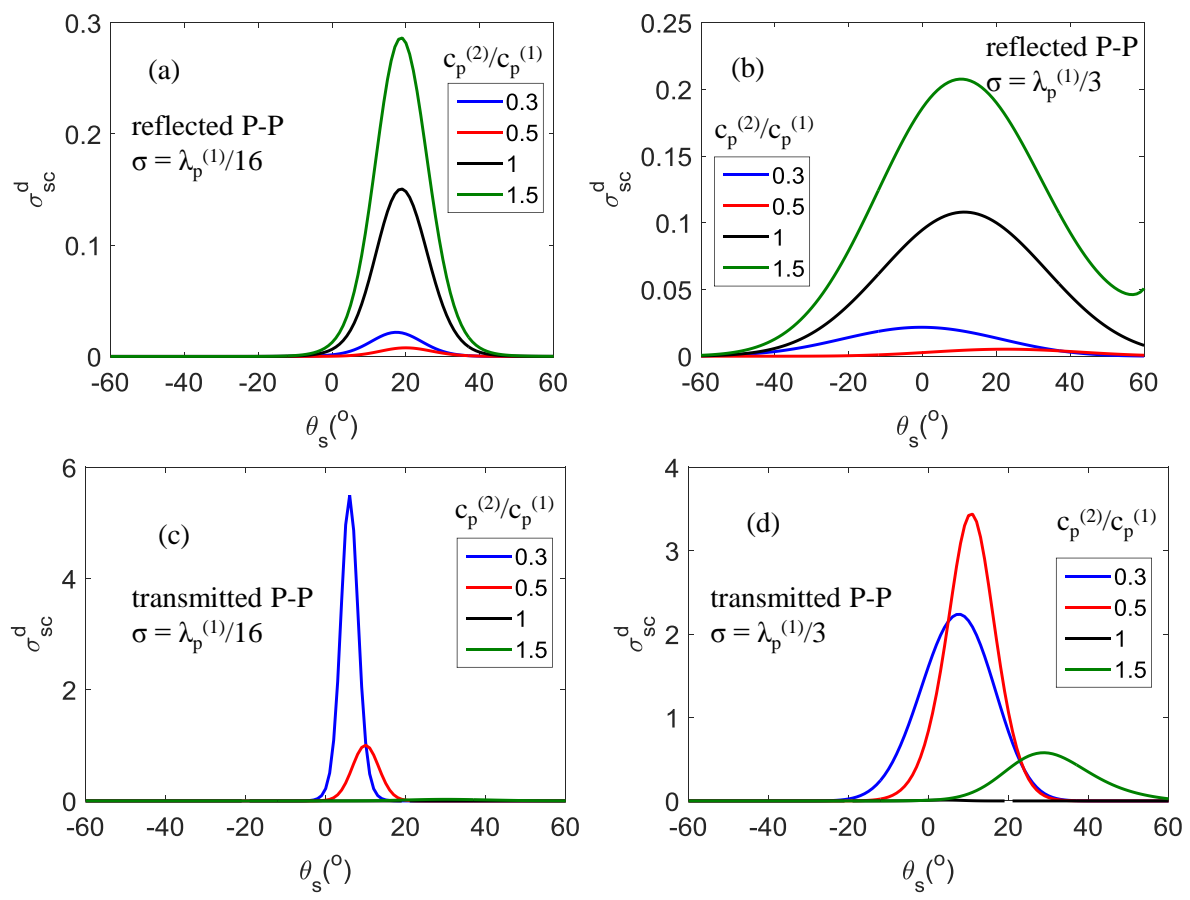

Figure 8: Diffuse scattering cross section $\left(\sigma_{s c}^{d}\right)$ from rough interfaces (Solid 1 is $\mathrm{Si}$; Solid 2 has the same density as Ge but various values of the wave speed.) by a $20^{\circ}$ incident $\mathrm{P}$ wave as a function of scattering angles. (a) Reflected P-P mode, $\sigma=\lambda_{p}^{(1)} / 16$. (b) Reflected P-P mode, $\sigma=\lambda_{p}^{(1)} / 3$. (c) Transmitted P-P mode, $\sigma=\lambda_{p}^{(1)} / 16$. (d) Transmitted P-P mode, $\sigma=$ $\lambda_{p}^{(1)} / 3$. The correlation length $\lambda_{0}=2 \lambda_{p}^{(1)}$.

both the reflection and transmission when $\sigma=\lambda_{p}^{(1)} / 8$. The theoretical results again match very well with those computed from numerical simulations for both $\mathrm{P}-\mathrm{P}$ and P-S modes, over a wide range of scattering angles. At grazing angles $\left(\left|\theta_{s}\right| \geq 70^{\circ}\right)$, the Kirchhoff approximation is not reliable according to previous studies Thorsos (1988); Shi et al. (2015); here the errors at these angles are not clearly seen in Fig. 5(c) and (d), due to the very small values of $\left\langle\sigma_{s c}>\right.$. Clear peaks at specular angles for different modes are found corresponding to the strong coherent field. In particular, the coherent peak is more dominant for the transmitted P-P mode in Fig. 5(d), than for the reflection in Fig. 5(c).

According to Eq. 13), the value of $C_{\beta}$ is expressed differently depending on whether it is a reflection or a transmission event. This difference of $C_{\beta}$ results in the divergence of the parameter $g_{\beta}$ in Eq. 25), which determines the attenua255 tion rate of the coherent field in terms of the roughness. For the Si/Ge interface simulated here, $g_{\beta}$ for the transmission is smaller than that for the reflection, hence contributing to a much slower attenuation of the coherent field for the 
transmission. Therefore a more noticeable peak is seen in Fig. 5(d), indicating that the coherent effects dictate the transmission. Similar conclusions are found for waves incident from liquid to solid using the phase screen method to model the coherent field Nagy and Rose (1993). Note that opposite situations exist where the transmitted coherent field decays quicker than the reflection. Such cases occur when $g_{\beta}$ for the transmission is larger than the value for the reflection in the specular direction in Eq. 25), indicating waves impinging from a hard material to a comparably very soft material. In addition, the divergence of $C_{\beta}$ in Eq. 13 also contributes to different behavior of the reflected and transmitted diffuse field, which will be shown in the next section.

\section{Physical analysis of the diffuse field}
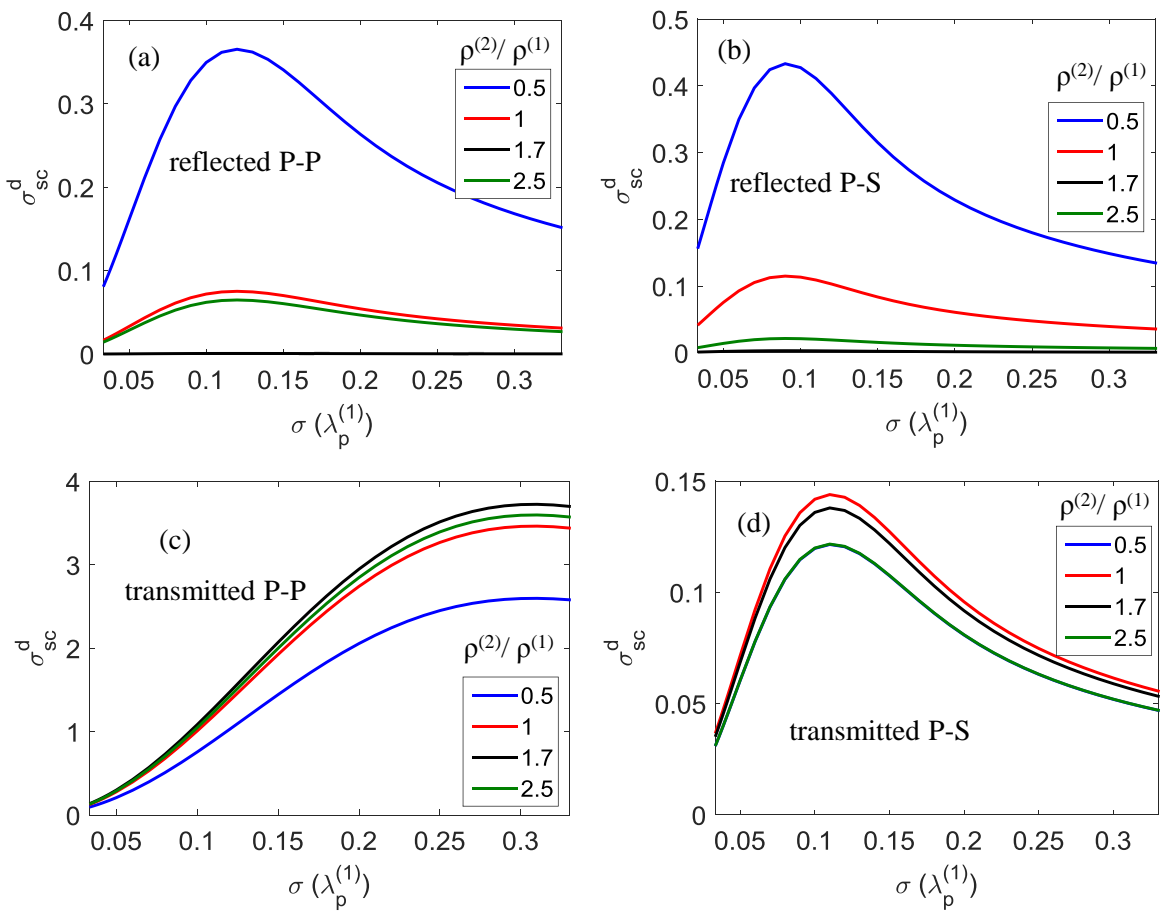

Figure 9: Diffuse scattering cross section $\left(\sigma_{s c}^{d}\right)$ at the specular direction from an interface (Solid 1 is $\mathrm{Si}$; Solid 2 has the same wave speed as Ge but various values of the density.) by a $20^{\circ}$ incident $\mathrm{P}$ wave as a function of rms values $(\sigma)$ when the correlation length $\lambda_{0}=2 \lambda_{p}^{(1)}$. (a) Reflected P-P. (b) Reflected P-S. (c) Transmitted P-P. (d) Transmitted P-S.

The scattering patterns of the diffuse field when a $\mathrm{P}$ wave is incident at $20^{\circ}$ from the side of Solid $1(\mathrm{Si})$ are shown in Fig. 6 for reflection and Fig. 7 for 
transmission. To explore the effect of material variation, Solid 2 is assumed to have the same wave speed as Ge, but with various values of the density (hence altering the elastic wave impedance); by changing the density, whilst keeping the wave speed of Solid 2, we are implicitly changing the Young's modulus. In each figure, we show results from a low $\left(\sigma=\lambda_{p}^{(1)} / 16\right)$ and a high roughness $(\sigma=$ $\left.\lambda_{p}^{(1)} / 3\right)$ case. The correlation length is assumed to be $\lambda_{0}=2 \lambda_{p}^{(1)}$ for both cases. As seen in Figs. 67, altering the density mainly changes the amplitude of $\sigma_{s c}^{d}$ for both reflected and transmitted waves, while having limited influence on the overall shape of the scattering pattern. From Eq. (27) we know that varying the density only affects the angular factor $\boldsymbol{F}_{\beta}$, and hence it weakly affects the shape of scattering patterns. A perfect match of the compressional wave impedance of the two solids (e. g. $\rho^{(2)} / \rho^{(1)}=1.7$ ) results in a zero reflection of the P-P 285 mode denoted as the black curve in Fig. 6(a) and (b), and correspondingly a maximum transmission amplitude in Fig. 7(a) and (b). In addition, the peaks of the scattering patterns in the low roughness cases are all located in the specular angles. This is because, in the small roughness approximation, the peak of the scattering pattern occurs when $A_{\beta}$ is zero from Eq. (28), corresponding to the specular angle.

The diffuse energy rises as the rms value increases from $\lambda_{p}^{(1)} / 10$ to $\lambda_{p}^{(1)} / 3$, altering the scattering patterns as shown in Figs. 6 and 7, the effect of the roughness is different depending on whether reflection and transmission is considered. In general the diffuse energy becomes more widely spread, and this trend is more apparent for the reflection than the transmission. In addition, the peaks of the reflection patterns are moving away from the specular direction in Fig. 6, while these peaks almost stay around the specular direction for the transmission shown in Fig. 7, especially for the transmitted P-P mode. The different behavior of the reflection and transmission patterns is explained using the high frequency asymptotic solution in Eq. (29). Recall that the 'specular' points for the reflection differ from those for the transmission as shown in Fig. 2, corresponding to different values of the surface slopes $\left(\partial h / \partial x_{1}=-A_{\beta} / C_{\beta}\right)$. For the $\mathrm{Si} / \mathrm{Ge}$ interface analysed here, the probability function $p_{g}\left(\partial h / \partial x_{1}\right)$ evaluated at the 'specular' points for the transmission has a much narrower angular distribution around the specular direction than it does for the reflection. As a consequence, the shape and also the peaks of the transmission pattern appear to be weakly affected by increasing $\sigma$, although the amplitude has been significantly changed.

We also investigate cases where we vary the wave speed $\left(c_{p}^{(2)}\right)$ of Solid 2 as shown in Fig. 8, while the density of Solid 2 is the same as Ge. Both the amplitude and the shape of the scattering pattern for the P-P mode are affected by the change of $c_{p}^{(2)}$, which is more apparent for the transmission case. It is also noticeable that the diffuse field for the transmitted P-P mode vanishes when $315 c_{p}^{(1)}=c_{p}^{(2)}$ as shown in Fig. 8(c). At this specific ratio of wave speed, from Eq. 113 we know that the parameter $C_{\beta}$ for the transmitted P-P mode is zero at the specular direction; the exponential decaying factor $g_{\beta}$ becomes zero from 

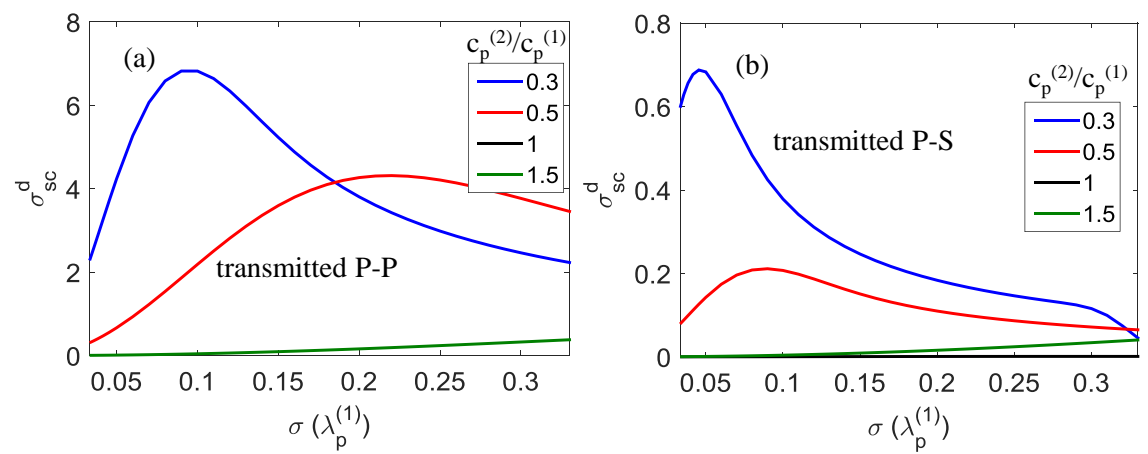

Figure 10: Diffuse scattering cross section $\left(\sigma_{s c}^{d}\right)$ at the specular direction from an interface (Solid 1 is $\mathrm{Si}$; Solid 2 has the same density as Ge but various values of wave speed.) by a $20^{\circ}$ incident $\mathrm{P}$ wave as a function of rms values $(\sigma)$ when the correlation length $\lambda_{0}=2 \lambda_{p}^{(1)}$. (a) Transmitted P-P. (b) Transmitted P-S.

Eq. 25), indicating that the transmission is completely occupied by the same coherent effect as that from a flat surface. Therefore the diffuse field for the transmission vanishes when $c_{p}^{(1)}=c_{p}^{(2)}$, in other words, the waves travel through the interface as if the 'roughness' did not exist if there is a perfect match of the wave speed.

\subsection{Specular scattering}

In many applications specific interest focusses on the interface scattering around the specular direction. For example, in the study of the phonon transport the specularity is a key parameter to calculate the thermal conductivity Maznev (2015); Hertzberg et al. (2014). Fig. 9 shows the specular scattering cross section $\left\langle\sigma_{s c}^{d}\right\rangle$ as a function of $\sigma$ when varying the density $\rho^{(2)}$. Clear peaks are observed at intermediate values of roughness $\sigma_{m e d}$, and for each mode the locations of these peaks are independent of the density $\rho^{(2)}$. It is noticeable that the peak for the transmitted P-P mode appears at a much higher roughness $\left(\sigma=\lambda_{p}^{(1)} / 4\right)$ than for other modes $\left(\sigma=\lambda_{p}^{(1)} / 8\right)$.

To understand $\sigma_{m e d}$ and the corresponding peaks, the Rayleigh parameter is quoted here Ogilvy (1991):

$$
R_{a}=\frac{\sqrt{g_{\beta}}}{2}
$$

where $g_{\beta}$ is from Eq. (25). The expression for $R_{a}$ differs from that in Ogilvy (1991), as we now include mode conversion and transmission. The Rayleigh criterion states that if $R_{a}$ is smaller than $\pi / 4$, the surface is relatively 'smooth', otherwise it is 'rough'. Here we let $R_{a}$ equal $\pi / 4$ and calculate the critical $\sigma$ values and find that they are almost the same as $\sigma_{\text {med }}$ observed from Fig. 9 


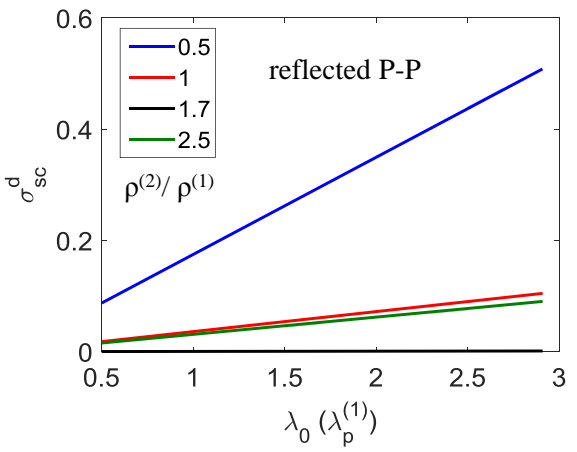

Figure 11: Diffuse scattering cross section $\left(\sigma_{s c}^{d}\right)$ for the reflected P-P mode at the specular direction from an interface (Solid 1 is $\mathrm{Si}$; Solid 2 has the same wave speed as Ge but various values of the density.) by a $20^{\circ}$ incident $\mathrm{P}$ wave as a function of correlation length $\left(\lambda_{0}\right)$ when the rms value $\sigma=\lambda_{p}^{(1)} / 10$.

for all the modes. The underlying physics in this are that the peak appears at the intermediate $\sigma_{m e d}$, at which the surface starts to transit from 'smooth' to 'rough' according to the Rayleigh criterion.

In addition, we plot the specular transmission in Fig. 10 when only varying the wave speed $c_{p}^{(2)}$ of Solid 2. The trends as increasing $\sigma$ are somewhat different from those when varying the density $\rho^{(2)}$ shown in Fig. 8 . For instance, the value of $\sigma_{\text {med }}$ increases when $c_{p}^{(2)} / c_{p}^{(1)}$ becomes larger, and it can still be accurately estimated by the Rayleigh criterion.

The role of the correlation length $\lambda_{0}$ on the specular scattering is also studied as shown in Fig. 11. The rms value is fixed as $\sigma=\lambda_{p}^{(1)} / 10$ in this case. As can be seen there exists a linear dependence of the specular scattering on $\lambda_{0}$. In the specular direction the parameter $A_{\beta}$ is zero and hence the exponential terms in Eq. (27) vanish, which clearly leads to the linear relationship between $355 \lambda_{0}$ and $\sigma_{s c}^{d}$. It should be noted that the simple linear dependence is found within the valid region of the Kirchhoff approximation Shi et al. (2015). For weakly correlated surfaces ( $\lambda_{0}$ is very small) the linearity might break down, and it is argued to be caused by the energy converted from the surface waves Maznev (2015).

\section{Conclusions}

In this article, we have derived theoretical formulae to predict the diffuse field of elastic waves scattered and transmitted by rough solid-solid interfaces, 
by using the Kirchhoff approximation. Theoretical solutions are evaluated by comparison with numerical Monte Carlo simulations, and the theory is shown to be accurate for surfaces with rms value $\sigma$ up to $\lambda / 3$. The new capability to model the diffuse field over a much wider range of roughness, beyond the limit of the small perturbation theory, enables the investigation of how interface roughness affects scattering when the rms value is large. Generally speaking, the 370 reflection and transmission are found to have different dependence on the interface roughness, and also a strong dependence on the material properties. As expected, the impedance mismatch plays an important role in determining the diffuse scattering, while it is found that the effects from the mismatch of density and wave speed are different. For the $\mathrm{Si} / \mathrm{Ge}$ interface evaluated here, the transmission of waves from $\mathrm{Si}$ to Ge is much less affected by the interface roughness than the reflection. As a result, the reflection diffuse energy tends to be more widely spread, and a shift of the peak of the reflection pattern away from the specular direction can be observed for interfaces with high roughness. In the extreme case when the two solids have the same wave speed, the transmission is only coherent and it is the same as that travelling through a flat interface. In addition, there exists an intermediate value of rms at which the diffuse energy in the specular direction reaches its maximum, and this rms value coincides with that estimated by the Rayleigh criterion, indicating that the conventional Rayleigh criterion is a precise measure of the transition point when the interface becomes 'rough'. It is also found that at the specular direction the scattering energy linearly depends on the correlation length within the valid region of the Kirchhoff approximation.

Most current studies in phonon transport are based either on Ziman's formulae only modelling the coherent field, or the so-called diffuse mismatch model, assuming a perfect rough surface that scatters waves to any direction with an equal probability, for the diffuse field. The formulae derived here provide a strong analytical tool that connects these hypothesis in the two extreme cases (extremely small and extremely large roughnesses). Therefore the new theory

395 would be very useful to study the elastic wave phonon boundary scattering, and hopefully to assist interpreting experimental phenomena of thermal transport in nanostructures Hertzberg et al. (2014); Cuffe et al. (2013) which are not well understood.

\section{References}

\section{References}

Achenbach, J.D., 2003. Reciprocity in elastodynamics. Cambridge University Express, Cambridge, UK.

Aksamija, Z., Knezevic, I., 2010. Anisotropy and boundary scattering in the lattice thermal conductivity of silicon nanomembranes. Phys. Rev. B 82, 045319 
Berry, M.V., 2015. Nature's optics and our understanding of light. Contemp. Phys. 56, 2-16.

Carrete, J., Gallego, L.J., Varela, L.M., 2011. Surface roughness and thermal conductivity of semiconductor nanowires: Going below the Casimir limit. Phys. Rev. B 84, 075403.

Chen, R., Hochbaum, A.I., Murphy, P., Moore, J., Yang, P., Majumdar, A., 2008. Thermal conductance of thin silicon nanowires. Phys. Rev. L 101, 105501.

Cuffe, J., Ristow, O., Chavez, E., Schepetov, A., Chapuis, P.O., Alzina, F., Hettich, M., Prunnila, M., Ahopelto, J., Dekorsy, T., Torres, C.M.S., 2013. Lifetime of confined acoustic phonons in ultrathin Silicon membranes. Phys. Rev. L 110, 095503.

Graff, K.F., 1975. Wave motion in elastic solids. Oxford University Press, Oxford, UK.

${ }_{420}$ Heron, J.S., Fournier, T., Mingo, N., Bourgeois, O., 2009. Mesoscopic size effects on the thermal conductance of silicon nanowire. Nano Lett. 9, 5 .

Hertzberg, J.B., Aksit, M., Otelaja, O.O., Stewart, D.A., Robinson, R.D., 2014. Direc measurements of surface scattering in Si nanosheets using a microscale phonon spectrometer: Implications for Casimir-limit predicted by Ziman theory. Nano Lett. 14, 403-415.

Huthwaite, P., 2014. Accelerated finite element elastodynamic simulations using the GPU. J. Comput. Phys. 257, 687-707.

Landau, L.D., Lifshitz, E.M., 1970. Theory of elasticity. Pergamon Press, Oxford, UK, 2nd ed.

430 Liang, Z., Sakikumar, K., Keblinski, P., 2014. Thermal transport across a subtrate-thin-film interface: Effects of film thickness and surface roughness. Phys. Rev. L 113, 065901.

Maznev, A.A., 2015. Boundary scattering of phonons: Specularity of randomly rough surface in the small-perturbation limit. Phys. Rev. B 91, 134306.

435 Maznev, A.A., Hofmann, F., Cuffe, J., Eliason, J.K., Nelson, K.A., 2015. Lifetime of high-order thickness resonances of thin silicon membranes. Ultrasonics $56,116-121$.

Nagy, P., Rose, J.H., 1993. Surface roughness and the ultrasonic detection of subsurface scatterers. J. Appl. Phys. 73, 566-580.

Ogilvy, J.A., 1986. Theoretical comparison of ultrasonic signal amplitudes from smooth and rough defects. NDT \& E Int. 19, 371-385. 
Ogilvy, J.A., 1991. Theory of Wave Scattering from Random Rough Surfaces. Adam Hilger Ltd., Bristol, UK.

Ogilvy, J.A., Culverwell, I.D., 1991. Elastic model for simulating ultrasonic inspection of smooth and rough defects. Ultrasonics 29, 490-496.

Ogilvy, J.A., Foster, J.R., 1989. Rough surfaces: gaussian or exponential statistics? J. Phys. D: Appl. Phys. 22, 1243-1251.

Pettit, J., Walker, A.E., Lowe, M.J.S., 2015. Improved detection of rough defects for ultrasonic nondestructive evaluation inspections based on finite element modeling of elastic wave scattering. IEEE Trans. Ultrason. Ferroelectr. Freq. Control. 62, 1797-1808.

Rajagopal, P., Drozdz, M., Skelton, E.A., Lowe, M.J.S., Craster, R.V., 2012. On the use of absorbing layers to simulate the propagation of elastic waves in unbounded isotropic media using commercially available finite element packages. NDT \& E Int. 51, 30-40.

Sadhu, J., Sinha, S., 2011. Room-temperature phonon boundary scattering below Casimir limit. Phys. Rev. B 84, 115450.

Schultz, C.A., Tokosz, M.N., 1994. Enhanced backscattering of seismic waves from a highly irregular random interface: P-SV case. Geophys. J. Int. 117, 783 .

Shi, F., Choi, W., Skelton, E.A., Lowe, M.J.S., Craster, R.V., 2014. A time domain finite element boundary integration method for ultrasonic nondestructive evaluation. IEEE Trans. Ultrason. Ferroelectr. Freq. Control 61, $2054-2066$.

${ }_{465}$ Shi, F., Choi, W., Skelton, E.A., Lowe, M.J.S., Craster, R.V., 2015. The validity of Kirchhoff theory for scattering of elastic waves from rough surfaces. Proc. R. Soc. A 471, 1-19.

Shi, F., Lowe, M.J.S., Xi, X., Craster, R.V., 2016. Diffuse scattered field of elastic waves from randomly rough surfaces using an analytical Kirchhoff theory. J. Mech. Phys. Solids 92, 260-277.

Sun, H., Pipe, K.P., 2012. Perturbation analysis of acoustic wave scattering at rough solid-solid interface. J. Appl. Phys. 111, 023510.

Thorsos, E.I., 1988. The validity of the Kirchhoff approximation for rough surface scattering using a Gaussian roughness spectrum. J. Acoust. Soc. Am. $83,78-92$.

Thorsos, E.I., Jackson, D.R., 1989. The validity of the perturbation approximation for rough surface scattering using a Gaussian roughness spectrum. J. Acoust. Soc. Am. 86, 261-277. 
Wang, Z., Alaniz, J.E., Jang, W., Garay, J.E., Dames, C., 2011. Thermal conductivity of nanocrystalline silicon: Importance of grain size and frequencydependent mean free paths. Nano Lett. 11, 2206-2213.

Weber, J., Sandmann, W., Dietsche, W., Kinder, H., 1978. Absense of anomalous Kapitza conductance on freshly cleaved surfaces. Phys. Rev. L 40, 22.

Wen, Y.C., Hsieh, C.L., Lin, K.H., Chen, H.P., Chin, S.C., Hsiao, C.L., Lin, Y.T., Chang, C.S., Chang, Y.C., Tu, L.W., Sun, C.K., 2009. Specular scattering probability of acoustic phonons in atomically flat interfaces. Phys. Rev. L 103, 264301.

Zhao, H., Freund, J.B., 2009. Phonon scattering at a rough interface between two fcc lattices. J. Appl. Phys. 105, 013515.

490 Ziman, J.M., 1960. Electrons and Phonons. Clarendon Press, Oxford, UK. 